\title{
Attualità in nefrologia pediatrica: le conoscenze di rilievo per il nefrologo dell’adulto
}

\author{
Alberto Edefonti ${ }^{1}$, Antonio Vergori ${ }^{2}$, Giovanni Montini ${ }^{1,3}$, Francesco Emma ${ }^{4}$ \\ ${ }^{1}$ UOC Nefrologia, Dialisi e Trapianto Pediatrico, Fondazione IRCCS Ca' Granda, Ospedale Maggiore Policlinico, Milano - Italy \\ ${ }^{2}$ UOC Pediatria, Ospedale Maggiore di Lodi, Lodi - Italy \\ ${ }^{3}$ Dipartimento di Scienze Cliniche e di Comunità, Università degli Studi di Milano, Milano - Italy \\ ${ }^{4}$ Dipartimento di Pediatrie Specialistiche - UOC di Nefrologia e Dialisi, Ospedale Pediatrico Bambino Gesù - IRCCS, Roma - Italy
}

\begin{abstract}
Updates in pediatric nephrology: relevant knowledge for the adult nephrologist
Examples of innovative research in pediatric nephrology include: a) the typically pediatric field of Congenital Anomalies of the Kidney and Urinary Tract (CAKUT), which has benefited from the discovery of numerous gene mutations responsible for the various malformations and the demonstration of the congenital origin of most of the renal damage, resulting in a decrease of invasive imaging, antibiotic prophylaxis and surgery;

b) the approach to glomerular diseases that appear in childhood, like idiopathic nephrotic syndrome (INS), IgA nephropathy (IgAN) and C3 glomerulopathies (C3G).

$B$ and T lymphocyte disregulations and molecular podocyte alterations of immunological and genetic origin have been described in INS as main determinants of proteinuria. In IgAN, the discovery of an abnormal IgA glycosilation in the mucosal B cells has driven to new trials with Budesonide and Sparsentan and to innovative therapies, like atacicept. A new classification of C3G has been proposed after the description of genetic mutations of factors inhibiting activation of the alternative complement pathway, and monoclonal anti-C5 antibody Eculizumab has consequently entered the therapeutic armamentarium;

c) the initial attempts at gene therapy, with promising results obtained in Alport syndrome, nephropathic cystinosis and Dent syndrome.

Moreover, a clear example of precision medicine is represented by the refinement of the dosage of Eculizumab in the treatment of atypical HUS, while slow-medicine recommendations exist for common clinical conditions, like urinary tract infections, microscopic hematuria and proteinuria.

Finally, a non-negligible series of guidelines and clinical practice recommendations have been developed. Those regarding CAKUT and glomerulopathies, here summarized, constitute the basis for a better understanding of the novelties described above.
\end{abstract}

Keywords: Pediatric guidelines, Pediatric kidney disease, Precision medicine, Slow-medicine

\section{Introduzione}

La crescita delle conoscenze e del numero degli articoli pubblicati in nefrologia pediatrica negli ultimi anni è stata esponenziale.

Received: February 22, 2021

Accepted: April 25, 2021

Published online: May 12, 2021

Indirizzo per la corrispondenza:

Alberto Edefonti

UOC Nefrologia, Dialisi e Trapianto Pediatrico

Fondazione IRCCS Ca' Granda

Ospedale Maggiore Policlinico, Milano

Via della Commenda 9

20122 Milano - Italy

aedefonti@hotmail.com
In questo processo, la nefrologia pediatrica ha seguito il percorso di altre specialità, beneficiando dapprima degli sviluppi della genetica e della biologia molecolare (1), poi, più recentemente, dell'intelligenza artificiale applicata ai grandi numeri (2) e, da ultimo, della disponibilità di terapie, quali gli anticorpi monoclonali, le cellule staminali e la terapia genica, che hanno aperto nuove prospettive per malattie considerate incurabili.

La comunicazione scientifica in nefrologia pediatrica si è mossa, quindi, tra due poli complementari: da un lato, la ricerca sui meccanismi fisiopatologici di malattie renali tuttora non chiarite dal punto di vista eziopatogenetico e, dall'altro, la sistematizzazione delle conoscenze acquisite, sotto forma di Linee Guida, raccomandazioni di pratica clinica e "consensus statements".

A sua volta, l'approccio clinico ha contemplato modalità apparentemente antitetiche, ma complementari, considerando 
sia la medicina di precisione che la slow-medicine. Queste si applicano a uno spettro di malattie per molti aspetti differenti rispetto a quelle incontrate nella nefrologia dell'adulto, dal momento che si riscontra una prevalenza di nefropatie congenite ed ereditarie, come, per esempio, le anomalie congenite del rene e delle vie urinarie (CAKUT), rispetto a quelle acquisite, e una bassa incidenza di comorbidità cardiovascolari e metaboliche. Inoltre, anche nel caso in cui la malattia renale sia la medesima, l'esordio clinico spesso precoce fa sì che la diagnosi e il trattamento iniziali siano comunque campo d'azione del nefrologo pediatra.

Da ultimo, come in altre specialità, la considerazione di rilevanti aspetti etici è stata, in questi ultimi anni, una cifra costante della comunicazione in nefrologia pediatrica.

\section{La ricerca sui meccanismi fisiopatologici e sulle terapie innovative}

\section{Le anomalie congenite del rene e delle vie urinarie}

Le anomalie congenite del rene e delle vie urinarie (CAKUT) comprendono un ampio spettro di malattie, dalla rara agenesia renale bilaterale, incompatibile con la vita, a condizioni più frequenti e meno gravi, quali la pelvi bifida o il reflusso vescico-ureterale.

Circa un quarto dei difetti congeniti riscontrati alla nascita appartiene all'apparato uropoietico e, a differenza di quanto si riscontra nell'adulto, oltre il $60 \%$ dei casi di insufficienza renale cronica in età pediatrica vede la propria causa nelle CAKUT.

Prima degli anni 2000, non ne era nota l'eziopatogenesi: forme familiari, anche se con quadri clinici eterogenei, facevano supporre fattori genetici, ma erano riconosciuti anche fattori ambientali, quali il diabete materno, l'uso di farmaci (per esempio, ACE inibitori) o carenze alimentari (vitamina A) durante la gravidanza. Negli ultimi due decenni sono state individuate, come cause di forme isolate di CAKUT, mutazioni di oltre 40 geni implicati nei processi della nefrogenesi e oltre 200 mutazioni sono state associate a forme sindromiche. Tuttavia, la maggior parte delle forme isolate non trova tuttora una causa precisa (1).

In questi anni, è anche emerso chiaramente che il danno renale associato alle uropatie non ostruttive e, tra queste, tipicamente a quella più conosciuta e ricercata dai pediatri, il reflusso vescico-ureterale, è molto più frequentemente congenito che acquisito e, in definitiva, secondario alle succitate mutazioni genetiche. In questo contesto, pertanto, le infezioni febbrili delle vie urinarie, che rappresentano l'espressività clinica del reflusso e che ne permettono la diagnosi, raramente portano da sole a un grave danno renale cronico. Di conseguenza, si è verificata, nell'ultimo decennio, una progressiva riduzione dell'aggressività medica, sia diagnostica, per quanto riguarda la prescrizione di cistografia minzionale e scintigrafia renale, che terapeutica, con un minore utilizzo della profilassi antibiotica a lungo termine e un minore ricorso alla chirurgia del reflusso $(2,3)$.
La ricerca nel campo delle nefro-uropatie malformative sta procedendo, con l'identificazione di numerosi nuovi geni causativi di malattia, grazie all'uso sempre più esteso delle nuove tecniche di sequenziamento, ma la strada appare piuttosto complessa, per la profonda eterogeneità, la variabile espressività e la penetranza incompleta delle varie forme di anomalie nefro-urologiche congenite.

Oltre allo studio dei geni implicati nel processo di nefrogenesi, un nuovo promettente campo di ricerca è rappresentato dallo studio dei geni espressi dalle cellule uroteliali che rivestono le vie urinarie (4). Dati recenti dimostrano un ruolo critico delle interazioni tra l'urotelio e il sottostante mesenchima nello sviluppo del tratto urinario: un disturbo di questo costante dialogo tra i due tipi cellulari produrrebbe variabili fenotipi di CAKUT.

La placca uroteliale ha anche un ruolo protettivo in risposta a eventi infiammatori e a ostruzioni del tratto urinario. Per tale motivo, sono allo studio nuovi biomarker urinari che hanno origine dalle cellule uroteliali, quali IL5 e CCL11, che potrebbero aiutare a selezionare i pazienti con ostruzione, come, per esempio, quelli affetti da stenosi del giunto pieloureterale, che richiedono una terapia medica o chirurgica, e a precisare meglio la loro prognosi.

Un altro utile biomarker, anch'esso espressione di interessamento uroteliale e recentemente rivisitato, è rappresentato dal grado di ispessimento della pelvi renale, che è in grado di aumentare notevolmente la sensibilità dell'ecografia nel predire la presenza di reflusso di alto grado nei bambini con idronefrosi o infezione delle vie urinarie $(5,6)$.

\section{Meccanismi patogenetici e terapia delle glomerulopatie}

Le lesioni renali che caratterizzano le malattie glomerulari del bambino sono state descritte per la maggior parte tra gli anni '60 e '70 del secolo scorso. Gli importanti progressi nel campo dell'interpretazione della biopsia renale, resi possibili dall'introduzione di tecniche innovative di immunoistochimica e dall'avvento della microscopia elettronica, hanno, infatti, permesso di definire dettagliatamente le caratteristiche cliniche e istologiche della malattia a lesioni minime, della glomerulosclerosi focale e segmentale e delle glomerulonefriti a depositi di IgA, membranosa e membranoproliferativa. Di conseguenza, è stato anche possibile stabilirne le principali correlazioni anatomo-cliniche, con importanti risvolti nella pratica clinica quotidiana.

Tuttavia, le basi eziopatogenetiche di molte glomerulopatie restano ancora oggi poco chiare, nonostante gli enormi progressi a cui abbiamo assistito in campo immunologico e genetico. Riassumiamo qui di seguito riscontri della recente letteratura che riguardano la malattia a lesioni minime, la glomerulosclerosi focale, la nefropatia da IgA e le glomerulonefriti a depositi di C3.

Quando la sindrome nefrosica idiopatica esordisce in bambini di età compresa tra 1 e 10 anni, presenta come correlato istologico una malattia a lesioni minime nel 70\%-90\% dei casi (7). La sindrome nefrosica è caratterizzata, dal punto di vista clinico, dalla comparsa di una proteinuria massiva, che 
determina un'ipoalbuminemia, con conseguente comparsa di deplezione intravascolare ed edema. La prognosi di questa condizione è generalmente buona, in quanto la risposta alla terapia steroidea porta alla risoluzione del quadro clinico nell' $85-93 \%$ dei casi. Tuttavia, il rischio di un elevato numero di recidive porta a un impatto sfavorevole sulla qualità di vita di questi bambini e delle loro famiglie e richiede, nella maggioranza dei casi, l'avvio di uno o più cicli di terapia immunosoppressiva.

La biopsia renale dei bambini con malattia a lesioni minime presenta un'istologia normale in microscopia ottica, da cui il nome della glomerulopatia. In microscopia elettronica, invece, si evidenzia un'estesa fusione dei pedicelli dei podociti, secondaria a una destrutturazione del citoscheletro di actina che ne sottintende la complessa architettura.

La genesi immunomediata o infiammatoria di questa malattia è testimoniata dalla risposta alla terapia corticosteroidea e immunosoppressiva. Negli ultimi vent'anni, inoltre, numerosi studi hanno suggerito un'azione sinergica di disregolazioni a carico dei linfociti B e T e di alterazioni podocitarie nel determinare esordio e recidiva di malattia (8).

A questo proposito, è stata recentemente osservata, nei pazienti con sindrome nefrosica trattati con l'anticorpo antiCD20 Rituximab, una forte correlazione tra recidiva di malattia e ricomparsa di cellule B di memoria, ponendo l'attenzione sui meccanismi di memoria immunologica nel determinismo della sindrome nefrosica (9).

Come accaduto per altre malattie che coinvolgono il sistema immunitario, anche in questo caso, studi genetici di popolazioni (GWAS) hanno evidenziato geni predisponenti alla comparsa di una sindrome nefrosica cortico-sensibile. Si tratta soprattutto di geni localizzati a livello del sistema maggiore di istocompatibilità (HLA) e di geni implicati nella regolazione del sistema immunitario (8).

La componente genetica succitata e quella ambientale, sotto forma di infezioni che scatenano l'attivazione del sistema immunitario, concorrono, quindi, a determinare l'esordio clinico e le recidive di proteinuria nei bambini con sindrome nefrosica cortico-sensibile.

Molti aspetti della patogenesi di questa malattia restano, tuttavia, ancora oscuri, il che spiega perché la sindrome nefrosica idiopatica rimanga uno degli ambiti di ricerca di maggiore interesse in nefrologia pediatrica.

Sfortunatamente, una percentuale variabile tra il $7 \%$ e il $15 \%$ dei bambini affetti da nefrosi idiopatica non risponde, all'esordio o in corso di successive recidive, alla terapia corticosteroidea standard, configurando un quadro clinico di cortico-resistenza. In tali frangenti, il quadro bioptico è spesso rappresentato da una glomerulosclerosi focale e segmentale e l'evoluzione, nonostante la terapia immunosoppressiva, anche con i recenti anticorpi monoclonali, è verso lo sviluppo di un'insufficienza renale terminale in circa la metà dei casi (10).

Studi genetici susseguitisi nell'ultimo ventennio hanno, in realtà, dimostrato la presenza di mutazioni (circa 40) a carico di proteine strutturali del podocita nel 30\% dei corticoresistenti, il che spiega la mancata risposta di tali casi alla terapia immunosoppressiva $(11,12)$. Nel restante $70 \%$ dei casi, il meccanismo che sottintende la comparsa di malattia non è stato ancora chiarito, sebbene esistano forti evidenze a favore del meccanismo immunomediato, quali l'elevato tasso di recidiva di glomerulosclerosi focale sul rene trapiantato (13). È ipotizzabile, quindi, la presenza di uno o più fattori di permeabilità circolante che destabilizzano la membrana podocitaria, causando la comparsa di sindrome nefrosica. L'identificazione dei fattori circolanti rappresenta uno degli obiettivi più importanti nel campo della ricerca in nefrologia pediatrica, in quanto permetterebbe di sviluppare terapie specifiche per questa malattia e di prevenire lo sviluppo di insufficienza renale nei bambini affetti.

La nefropatia da depositi di IgA è una glomerulonefrite ad andamento cronico progressivo, che determina lo sviluppo di insufficienza renale terminale nel $30 \%$ dei casi dopo 20 anni di malattia $(14,15)$ e rappresenta la glomerulonefrite cronica più diffusa al mondo, con 30 casi per milione di abitanti (14). Dal momento della scoperta, nel 1964, a oggi sono stati compiuti numerosi progressi nella comprensione dei meccanismi patogenetici alla base della sua insorgenza. Un'anomala glicosilazione a carico delle IgA, la formazione di immunocomplessi IgA circolanti, un'attivazione anomala della via delle leptine del complemento e la presenza di anomalie dell'immunità mucosale costituiscono i dati di maggiore rilievo. Tuttavia, l'acquisizione di queste conoscenze non ha avuto un impatto significativo sulla cura dei pazienti; l'approccio terapeutico attuale si basa su evidenze risalenti agli anni ' 80 e '90, periodo in cui è stata provata l'efficacia parziale di coticosteroidi e inibitori del sistema renina-angiotensina nel rallentare la progressione della malattia.

Nuove terapie sono, peraltro, alle porte. Risultati promettenti si stanno ottenendo con I'utilizzo della Budesonide, steroide con attività selettiva sulla mucosa intestinale (16), e con lo Sparsentan, che agisce sia sul sistema renina-angiotensina che su quello dell'endotelina (studio PROTECT).

Sono stati anche avviati diversi studi, attualmente in fase preclinica, sull'utilizzo di farmaci innovativi che mirano a inibire selettivamente $i$ linfociti B che producono le IgA con alterata glicosilazione $\mathrm{O}$, in un altro caso, a rimuovere selettivamente le IgA dal glomerulo mediante I'utilizzo di proteasi (17).

L'utilizzo di Rituximab, contrariamente a quanto ipotizzabile, si è rivelato inefficace nel contrastare la progressione della malattia, probabilmente perché il farmaco non è attivo sulle plasmacellule localizzate a livello della mucosa intestinale, primariamente responsabili della produzione delle IgA deglicosilate (18). È proprio su queste cellule che si sta concentrando la ricerca più recente, nel tentativo di impedirne la maturazione mediante l'inibizione dei fattori BAFF e PRIL (studio sull'efficacia dell'inibitore di APRIL Atacicept, studio BRIGHT-SC). 
Sempre in quest'ambito, un ulteriore campo di ricerca è rappresentato dal sistema del complemento: sono stati, infatti, ottenuti risultati promettenti con diverse molecole che agiscono inibendo l'attività della frazione C3 e/o C5 $(17,19)$.

Da quanto detto, si evince che sarà necessario attendere alcuni anni prima di avere a disposizione dei farmaci in grado di modificare la storia naturale della nefropatia da IgA.

La glomerulonefrite da depositi di C3 è stata descritta per la prima volta negli anni '70 e, per oltre 30 anni, la sua patogenesi è rimasta sconosciuta. A partire dal 2007, anno in cui sono state descritte le prime mutazioni a carico delle proteine regolatrici del sistema del complemento, questa malattia è diventata l'esempio della medicina traslazionale.

In poco più di 10 anni, infatti, sono state descritte numerose mutazioni a carico dei geni regolatori della via alterna, che ne favoriscono un'attivazione spontanea e incontrollata. Nelle forme in cui non è possibile riscontrare alterazioni genetiche, spesso è identificabile una causa autoimmunitaria, vale a dire la presenza di anticorpi diretti contro proteine regolatrici.

La ricerca in quest'ambito ha, infine, permesso di stabilire l'esistenza di un continuum tra la glomerulonefrite da depositi di C3, in cui il sistema del complemento è attivo principalmente in fase fluida, e la sindrome emolitico-uremica atipica, in cui l'attività del complemento appare incontrollata in prossimità degli endoteli (20).

La comprensione della patogenesi della glomerulonefrite da depositi di C3 ha permesso di mettere a punto strategie terapeutiche in grado di contrastare la progressione della malattia verso l'insufficienza renale.

Nelle forme anticorpo-mediate, la terapia consiste nell'utilizzo di plasmaferesi associata a Rituximab (21).

Nei casi secondari a mutazioni genetiche, invece, l'unico farmaco disponibile per la cura è l'Eculizumab (anti-C5a), che risulta, peraltro, efficace solo in una parte dei pazienti (22). Ciò è dovuto al fatto che, nella maggior parte di questi casi, l'attivazione della cascata del complemento avviene a monte di C5, vale a dire a carico della C3 convertasi $(20,22)$. Per questa ragione, sono, attualmente, in studio molecole in grado di inibire direttamente la C3 convertasi, il fattore B o il fattore D (9).

Identificare una terapia efficace e duratura per queste glomerulonefriti croniche rappresenta, pertanto, un obiettivo importante, sia per i nefrologi pediatri che per quelli dell'adulto, che "ricevono in eredità" questi pazienti al termine dell'adolescenza.

\section{La terapia genica delle malattie renali}

$\grave{E}$, oggi, ben chiaro che le nefropatie genetiche si manifestano e vengono diagnosticate nella maggioranza dei casi già in età pediatrica e rappresentano, quindi, tradizionalmente, un settore di interesse specifico del nefrologo pediatra. Nonostante ciò, la terapia genica costituisce un campo relativamente ancora poco avanzato. Ciò è dovuto essenzialmente alla complessità del tessuto renale, che rende il "targeting" di specifiche cellule particolarmente complesso, alla scarsa accessibilità delle cellule podocitarie, esposte principalmente sul versante urinario, e al ridotto numero di precursori staminali renali che potrebbero rigenerare tessuto sano dopo correzione genica.

Alcuni studi, volti a superare questi ostacoli, sono, tuttavia, in corso. Il primo riguarda la sindrome di Alport, caratterizzata, come è noto, da diverse mutazioni delle catene del collagene di tipo IV, che causano uno slaminamento della membrana basale glomerulare, con progressiva perdita dell'integrità dei glomeruli e danno renale cronico. La terapia genica è, in questo caso, complicata dalle grandi dimensioni dei geni del collagene. Gli attuali approcci cercano, quindi, di preservare un'espressione parziale delle catene mutate, tramite "exone skipping" o "chaperon", nell'intento di garantire un'espressione residua, che conservi solo in parte l'integrità della membrana basale glomerulare, ma che sia comunque in grado di prolungare la sopravvivenza renale (23).

Un secondo stimolante campo di ricerca riguarda il trapianto di cellule staminali ematopoietiche nelle tubulopatie genetiche. II migliore esempio a questo proposito è rappresentato dalla cistinosi nefropatica, che è caratterizzata, come è noto, dall'accumulo di cistina nei lisosomi e, clinicamente, dalla comparsa, sin dai primi mesi di vita, di una grave tubulopatia prossimale (sindrome di Fanconi), che progredisce verso l'insufficienza renale terminale. Studi recenti hanno dimostrato che cellule ematopoietiche trapiantate hanno la capacità di migrare nell'interstizio renale, dove si differenziano in monociti-macrofagi e acquisiscono la capacità di creare dei "nano-tunnel", attraverso i quali possono trasferire organelli e materiale genetico alle cellule tubulari (24).

Lo stesso effetto è stato osservato sperimentalmente in un'altra malattia tubulare renale, la sindrome di Dent (25).

Su queste basi, è in corso uno studio clinico di autotrapianto di midollo in soggetti affetti da cistinosi nefropatica, dopo correzione ex-vivo con vettori virali della loro mutazione genetica (NCT03897361). Se si dovessero confermare i risultati incoraggianti ottenuti sull'animale, questi studi potrebbero aprire una nuova strategia terapeutica per diverse malattie tubulari renali clinicamente molto invalidanti e con prognosi sfavorevole.

\section{I due modelli complementari di attività clinica in nefrologia pediatrica}

All'interno della nefrologia pediatrica, come peraltro avvenuto anche in altre specialità, sono state in questi anni riconosciute due diverse modalità, tra loro complementari, di intervento diagnostico e terapeutico. La prima è la medicina di precisione, svolta principalmente nei centri specialistici di terzo livello e focalizzata sulle necessità diagnostiche e terapeutiche del singolo paziente, affetto generalmente da 
malattie rare; la seconda è rappresentata dalla slow-medicine, rivolta soprattutto ai pediatri e ai medici di famiglia, che ha come oggetto principale l'atteggiamento corretto verso i problemi nefro-urologici di più frequente riscontro nella pratica clinica, come, per esempio, le infezioni delle vie urinarie e le microematurie.

\section{La medicina di precisione}

Esistono oggi in letteratura diversi termini per definire l'approccio diagnostico e terapeutico individualizzato al singolo paziente, tra cui i più noti sono "medicina personalizzata", utilizzato soprattutto in Europa, e "medicina di precisione", di uso più frequente negli Stati Uniti. La medicina di precisione, termine che preferiamo per i maggiori riscontri in letteratura, è orientata in maniera specifica alle necessità del singolo paziente ed è stata definita dal Dottor Collins, Direttore dei National Institutes of Health degli Stati Uniti, come "I'insieme di strategie di prevenzione e trattamento che tengono conto della variabilità individuale".

L'emato-oncologia è la specialità che ha aperto la strada e che ha maggiormente utilizzato questo approccio. Uno dei primi esempi di terapia di precisione è stato rappresentato dall'uso dell'anticorpo anti-CD20, Rituximab, in alcuni linfomi e in alcune leucemie. La genomica ha, poi, permesso un affinamento del suo utilizzo, individuando indicatori di predisposizione, prognosi e, perfino, capacità di risposta a uno specifico schema di trattamento.

Anche in nefrologia pediatrica esistono numerosi studi indirizzati a sviluppare la medicina di precisione. Un esempio dell'importanza (per il paziente) e dell'utilità (per il sistema sanitario) di un trattamento individualizzato sulla base degli effettivi bisogni ci viene offerto dalla sindrome emolitica uremica atipica.

A partire dal 2009, si è reso disponibile nella pratica clinica un inibitore dell'attivazione delle vie del complemento, l'anticorpo monoclonale umanizzato anti-C5, Eculizumab, che ha radicalmente modificato l'evoluzione di questa grave patologia, portando il tasso di remissione, con funzione renale conservata, dal $30 \%$ a più dell' $80 \%$ (26). L'iniziale schema terapeutico prevedeva la somministrazione del farmaco ogni 14 giorni per tutta la vita, con un costo per paziente di circa 400.000 euro all'anno. Un'attenta calibrazione del trattamento, basata sul peso corporeo e sul monitoraggio della funzione globale del complemento, valutata con il dosaggio del $\mathrm{CH} 50$, ha consentito di dilazionare la somministrazione dell'inibitore del C5 a una volta al mese, con un miglioramento della qualità di vita dei pazienti e un dimezzamento dell'ingente costo iniziale $(27,28)$.

Inoltre, la corretta caratterizzazione, mediante biologia molecolare, dell'eziologia della malattia (che dipende da una disregolazione del complemento idiopatica, soprattutto di origine genetica, o secondaria, su base auto-anticorpale) ha reso possibile l'interruzione del farmaco in quasi la metà dei pazienti, con un ulteriore ingente risparmio di disagi, di esposizione al farmaco e ai suoi possibili effetti collaterali e di risorse (29).

Ha contribuito a questo importante risultato anche l'identificazione dello stick delle urine per emoglobinuria come facile e affidabile strumento per il monitoraggio a domicilio del paziente, al fine di diagnosticare eventuali recidive. La personalizzazione delle cure attraverso i succitati esami diagnostici ha, quindi, abbattuto i costi medi del trattamento per paziente a circa un quarto della spesa inizialmente prevista.

Altri esempi di medicina di precisione nel campo della nefrologia pediatrica, che hanno portato in questi anni a sviluppi significativi dal punto di vista della diagnosi e del trattamento, sono rappresentati dalla sindrome nefrosica idiopatica (30), dal trapianto di rene $(31,32)$ e dalla sindrome di Bartter (33), per i quali forniamo il riferimento bibliografico.

Come già accennato, l'elevato costo degli esami e delle terapie specifiche e l'elevata competenza richiesta fanno sì che la medicina di precisione sia appannaggio dei centri specializzati di nefrologia pediatrica.

\section{La slow-medicine}

I protocolli di pratica clinica e le Linee Guida sono fondati sulla medicina basata sull'evidenza, che segue un approccio orientato alla popolazione generale e valuta l'efficacia delle terapie su base statistica, favorendo la media nei confronti del caso singolo. Da essi derivano le raccomandazioni della slow-medicine sul corretto approccio iniziale alla diagnosi e alla cura di sintomi o di dati di laboratorio alterati, di origine renale, quando siano osservati negli ambulatori di pediatria di famiglia o in ospedali non specializzati.

La Società Italiana di Nefrologia Pediatrica ha aderito all'iniziativa del gruppo di Slow-Medicine, nell'ottica che molti esami e terapie, anche se ampiamente diffusi, non apportino benefici per i pazienti e che, talora, possano essere persino deleteri. In questa prospettiva, è stata formulata una serie di raccomandazioni riportate sul sito di Slow-Medicine (Online).

Qui ricordiamo solo che esse riguardano importanti problemi pratici, quali la diagnosi corretta di IVU, l'approccio alla proteinuria e alla microematuria, l'utilizzo dell'infusione di albumina nella sindrome nefrosica e, infine, l'enuresi.

II risparmio di disagi fisici e psicologici per il paziente e la famiglia e di costi per il Sistema Sanitario Nazionale appare del tutto evidente. Dal momento che è sempre indicato nelle raccomandazioni il momento in cui deve essere fatto riferimento allo specialista, viene comunque soddisfatto il principio della cura ottimale del paziente.

\section{La sistematizzazione delle conoscenze scientifiche}

L'esame della letteratura nefrologica degli ultimi anni rivela un interesse spiccato verso la pubblicazione di lavori che, seguendo regole ben codificate per l'impostazione dei 
quesiti e per la definizione del grado di raccomandazione, forniscano indicazioni oggettive di ordine diagnostico e/o terapeutico sulla gestione di diverse malattie renali e siano in grado di guidare le scelte cliniche di medici, anche se non esperti o residenti in paesi in via di sviluppo.

Al vantaggio di una diffusione delle conoscenze basilari nella comunità nefrologica pediatrica (e non solo), si aggiunge la possibilità di uniformare nel tempo le casistiche di diversi centri riguardo a definizione, modalità di diagnosi e trattamento iniziale di una malattia. Non ultima, la possibilità di accorpare pazienti e di disporre di coorti informative sufficientemente ampie da consentire un aumento della dimensione dei dati raccolti (tallone d'Achille della letteratura specialistica pediatrica) consente di sviluppare meglio trial clinici relativi a farmaci o ad altre modalità di trattamento.

Tuttavia, le malattie renali tuttora non coperte da Linee Guida o gli aspetti delle medesime non ancora chiariti sono molto più numerosi rispetto alle nefropatie e alle tematiche già oggetto di attenzione. Ciò riflette una difficoltà oggettiva nella stesura di Linee Guida e di raccomandazioni autorevoli, a causa della mancanza di studi validi, soprattutto randomizzati, su cui basarle. La maggioranza del materiale a disposizione degli autori è, infatti, costituita da studi monocentrici di coorte, con casistica limitata e spesso eterogenei tra di loro, che portano a gradi di raccomandazione inevitabilmente bassi.

Può, comunque, rappresentare uno strumento utile per il nefrologo dell'adulto una breve sintesi (Tab. I) delle raccomandazioni riguardanti le patologie glomerulari e le CAKUT, per le quali sono state esposte le principali novità nelle pagine precedenti. La Tabella riassume le conoscenze di base su cui vi è consenso in letteratura e contribuisce a una migliore comprensione degli sviluppi descritti.

A causa della spinta, favorita dalle stesse società scientifiche, verso una sempre maggiore globalizzazione delle conoscenze, le pubblicazioni inerenti Linee Guida e raccomandazioni di pratica clinica sono in crescita e rappresentano un utile punto di riferimento nella relazione tra nefrologo pediatra e dell'adulto, specie nel processo di transizione dei pazienti adolescenti verso i centri di nefrologia dell'adulto.

\section{Aspetti etici in nefrologia pediatrica}

Negli ultimi anni, si è palesato un drammatico aumento della frequenza dei casi di insufficienza renale cronica e acuta, soprattutto in età adulta e nei paesi a basso-medio reddito (low-medium income countries, LMIC) (34), causando un impatto economico e sociale devastante sui sistemi sanitari e sulla società civile di queste nazioni.

Inoltre, diversi lavori epidemiologici, nell'adulto come nel bambino, hanno sottolineato che fattori "non medici", in particolare socio-economici, culturali e relativi al livello di istruzione delle famiglie, contribuiscono alla comparsa e alla progressione di numerose malattie renali, influenzandone la morbilità e la mortalità (35-37). Tali fattori determinano in senso negativo i risultati finali delle cure mediche, non solo, come sembra intuitivo, nei paesi LMIC e BRICS (Brasile, India, Cina e Sud Africa) (38), ma anche a livello dei paesi ad alto reddito (high income countries, $\mathrm{HIC}$ ) in particolari strati della popolazione caratterizzati da povertà, basso grado di istruzione e isolamento sociale $(39,40)$.

Il fenomeno della disuguaglianza e dell'iniquità di trattamento (41) è stato riconosciuto dalle società scientifiche internazionali di nefrologia dell'adulto e del bambino (35), che hanno pubblicato documenti programmatici (42) e intrapreso iniziative concrete, mirate alla prevenzione e alla diagnosi precoce della CKD, al trattamento universale con dialisi e trapianto renale dello stadio finale della medesima e, più recentemente, alla diagnosi e alla terapia del danno renale acuto (Acute Kidney Injury, AKI) anche nel bambino.

TABELLA I - Linee guida per la diagnosi e il trattamento di patologie con esordio in età pediatrica, di interesse per il nefrologo dell'adulto.

\begin{tabular}{|c|c|}
\hline Glomerulopatie & Abstract \\
\hline $\begin{array}{l}\text { "The Italian Society for Pediatric Nephrology } \\
\text { (SINePe) consensus document on the man- } \\
\text { agement of nephrotic syndrome in children: } \\
\text { Part I - Diagnosis and treatment of the first epi- } \\
\text { sode and the first relapse" (45) } \\
\text { Uploaded on: SINePe* }\end{array}$ & $\begin{array}{l}\text { II trattamento iniziale della s. nefrosica idiopatica è rappresentato da prednisone per } \\
\text { os alla dose di } 60 \mathrm{mg} / \mathrm{m}^{2} / \text { die per } 6 \text { settimane, seguito da altre } 6 \text { settimane a } 40 \mathrm{mg} / \mathrm{m}^{2} \text { a } \\
\text { giorni alterni. } \\
\text { II trattamento della prima recidiva prevede prednisone alla dose di } 60 \mathrm{mg} / \mathrm{m}^{2} / \text { die fino } \\
\text { a } 5 \text { giorni dopo la scomparsa della proteinuria, seguito da } 4 \text { settimane a } 40 \mathrm{mg} / \mathrm{m}^{2} \text { a giorni } \\
\text { alterni. } \\
\text { Si definisce cortico-resistente una sindrome nefrosica che non abbia risposto alle } 4 \\
\text { settimane di prednisone giornaliero a dose piena, seguite da } 3 \text { boli di metilprednisolone } \\
\text { (500 mg/m²) e da altre } 2 \text { settimane a dose piena. } \\
\text { Indicazioni alla biopsia renale sono: } \\
\text { - steroido-resistenza; } \\
\text { - esordio prima dei } 12 \text { mesi o dopo i } 12 \text { anni di età; } \\
\text { - sospetto di forma secondaria. } \\
\text { Indicazioni all'esecuzione di indagini genetiche, oltre alla cortico-resistenza, sono: } \\
\text { - esordio nei primi } 12 \text { mesi di vita; } \\
\text { - familiarità per sindrome nefrosica; } \\
\text { - presenza di segni sindromici. }\end{array}$ \\
\hline
\end{tabular}




\begin{tabular}{|c|c|}
\hline Glomerulopatie & Abstract \\
\hline $\begin{array}{l}\text { "IPNA clinical practice recommendations for } \\
\text { the diagnosis and management of children with } \\
\text { steroid-resistant nephrotic syndrome" (46) } \\
\text { Uploaded on: IPNA** }\end{array}$ & $\begin{array}{l}\text { La prima linea di trattamento della s. nefrosica cortico-resistente (SNCR) è rappresenta- } \\
\text { ta dagli inibitori della calcineurina (CNIS), associati ad ACE-inibitori (ACEi) o a bloccanti dei } \\
\text { recettori dell'angiotensina (ARBS). Contestualmente, è opportuno avviare la diminuzione } \\
\text { progressiva del prednisone. } \\
\text { Le forme di SNCR su base genetica non beneficiano degli immunosoppressori, per cui, } \\
\text { non appena acquisita la diagnosi, è opportuno procedere alla loro sospensione, prose- } \\
\text { guendo la terapia con ACEi o ARBs. } \\
\text { Le forme immunomediate (non monogeniche) di SNCR si definiscono resistenti al trat- } \\
\text { tamento con CNIs dopo } 6 \text { mesi di trattamento a opportuno dosaggio e con adeguati livelli } \\
\text { ematici. } \\
\text { In questi casi, è opportuno eseguire tentativi di trattamento con farmaci risparmiatori } \\
\text { di steroidi (micofenolato mofetile, ciclofosfamide, rituximab). In caso di fallimento, si può } \\
\text { considerare l'utilizzo di Ofatumumab, plasmaferesi o immuno-adsorbimento. }\end{array}$ \\
\hline $\begin{array}{l}\text { "Expert Guidelines for the Management of Al- } \\
\text { port Syndrome and Thin Basement Membrane } \\
\text { Nephropathy" (47) } \\
\text { Uploaded on: ERKNet*** }\end{array}$ & $\begin{array}{l}\text { Sono descritti i criteri clinici per la diagnosi di sindrome di Alport e le diverse modalità } \\
\text { di trasmissione della malattia, insieme alle mutazioni del collagene IV responsabili di } \\
\text { ciascuna forma: X-linked (la più frequente), autosomica dominante, autosomica recessiva. } \\
\text { Si definiscono, poi, le caratteristiche cliniche della nefropatia da membrane basali sot- } \\
\text { tili e le relative mutazioni in eterozigosi a carico di COL4A4 o COL4A3, che configurano, } \\
\text { in realtà, uno stato di portatore di sindrome di Alport autosomica recessiva. } \\
\text { Dal momento che il gold standard per la diagnosi è rappresentato dall'indagine ge- } \\
\text { netica e che questa risulta fondamentale anche per stabilire la prognosi, tutti i membri } \\
\text { della famiglia di pazienti con sindrome di Alport X-linked devono essere indirizzati allo } \\
\text { studio genetico e posti in follow-up. } \\
\text { I maschi con malattia X-linked e tutti i pazienti con malattia autosomica recessiva devo- } \\
\text { no essere posti in terapia con ACEi o ARBs. } \\
\text { Alle madri di pazienti affetti da malattia X-linked viene sconsigliata la donazione del } \\
\text { proprio rene al figlio malato, data l'elevata probabilità per loro stesse di andare incontro } \\
\text { a IR terminale. }\end{array}$ \\
\hline $\begin{array}{l}\text { "An international consensus approach to the } \\
\text { management of atypical hemolytic uremic } \\
\text { syndrome in children" (48) } \\
\text { Uploaded on: ERKNet*** }\end{array}$ & $\begin{array}{l}\text { La sindrome emolitico-uremica atipica è causata, nel } 60-70 \% \text { dei casi, da disregolazioni a } \\
\text { carico della via alterna del complemento, su base ereditaria o acquisita. La prima linea di } \\
\text { trattamento è rappresentata dall'Eculizumab (anticorpo monoclonale anti-C5a), da iniziarsi } \\
\text { prima della conferma della mutazione genetica, in quanto il ritardo terapeutico aumenta il } \\
\text { rischio di progressione verso l'IR terminale. Qualora l'Eculizumab non risulti prontamente di- } \\
\text { sponibile, è indicata la plasmaferesi, che dovrebbe essere iniziata entro } 48 \text { ore dalla diagnosi. } \\
\text { Un ulteriore accertamento da eseguire in fase acuta, oltre allo studio genetico, è il } \\
\text { dosaggio degli anticorpi anti-fattore H, la cui positività prevede ulteriori possibilità tera- } \\
\text { peutiche (corticosteroidi, micofenolato mofetile, ciclofosfamide). }\end{array}$ \\
\hline $\begin{array}{l}\text { "Updated Italian recommendations for the } \\
\text { diagnosis, treatment and follow-up of the } \\
\text { first febrile urinary tract infection in young } \\
\text { children" (3) } \\
\text { Uploaded on: SINePe* }\end{array}$ & $\begin{array}{l}\text { Sono illustrati i criteri di diagnosi clinici e di laboratorio di IVU (p. es., urine e urinocoltu- } \\
\text { ra, con le modalità di raccolta sterile) nei bambini tra i } 2 \text { mesi e i } 3 \text { anni. } \\
\text { Dal momento che il germe maggiormente coinvolto è l'Escherichia coli, la terapia an- } \\
\text { tibiotica empirica di prima linea è rappresentata dall'amoxicillina + acido clavulanico } \\
\text { per os alla dose di } 100 \mathrm{mg} / \mathrm{kg} \text { /die. In assenza di criteri di ospedalizzazione, la terapia può } \\
\text { essere eseguita a domicilio. } \\
\text { In caso di infezioni gravi, sono consigliabili cefalosporine per via parenterale. La durata } \\
\text { del trattamento è di } 10 \text { giorni e, non appena le condizioni cliniche lo permettano, è possi- } \\
\text { bile passare alla terapia orale. } \\
\text { Gli accertamenti strumentali contemplano un'ecografia } 2-4 \text { settimane dopo il primo } \\
\text { episodio. Nel caso di alterazioni ecografiche o di infezione causata da germe diverso da E. } \\
\text { coli, è opportuno eseguire un'indagine di secondo livello per lo studio del RVU (cistogra- } \\
\text { fia minzionale o cistosonografia, scintigrafia renale diretta). } \\
\text { Nei maschi, la tecnica di scelta è rappresentata dalla cistografia minzionale, per per- } \\
\text { mettere lo studio dell'uretra. } \\
\text { La profilassi antibiotica dopo il primo episodio di UTI febbrile non è raccomandata, } \\
\text { salvo particolari indicazioni. }\end{array}$ \\
\hline $\begin{array}{l}\text { "AUA Guideline on Management of Primary } \\
\text { Vesicoureteral Reflux in Children" (49) } \\
\text { Uploaded on: ERKNet*** }\end{array}$ & $\begin{array}{l}\text { L'inquadramento di un bambino con reflusso vescico-ureterale (RVU) prevede, come in- } \\
\text { dagini preliminari, un esame delle urine e una creatininemia basale. In presenza di batte- } \\
\text { riuria, è indicata l'urinocoltura, anche in assenza di franche infezioni. } \\
\text { L'ecografia è la metodica di scelta per lo studio basale delle vie urinarie, eventualmente } \\
\text { seguita da una scintigrafia con DMSA, per valutare la funzione renale e l'eventuale pre- } \\
\text { senza di cicatrici. }\end{array}$ \\
\hline
\end{tabular}


TABELLA I - (Continua)

\begin{tabular}{|c|c|}
\hline Glomerulopatie & Abstract \\
\hline & $\begin{array}{l}\text { Un corretto inquadramento non può prescindere dallo studio delle abitudini minzion- } \\
\text { ali ed eventualmente della funzionalità vescicale, compatibilmente con l'età. } \\
\text { La profilassi antibiotica deve essere presa in considerazione nei bambini con RVU di } \\
\text { età inferiore a un anno con storia di IVU febbrili ricorrenti. } \\
\text { Nei bambini più grandi, di fondamentale importanza risulta la correzione di abitudini } \\
\text { minzionali errate e dell'instabilità vescicale. } \\
\text { L'intervento chirurgico va considerato nei bambini che continuano a presentare infe- } \\
\text { zioni in presenza di una funzionalità vescicale accettabile. }\end{array}$ \\
\hline $\begin{array}{l}\text { "Multidisciplinary Consensus on the Classifica- } \\
\text { tion of Prenatal and Postnatal Urinary Tract } \\
\text { Dilation" (50) } \\
\text { Uploaded on: ERKNet*** }\end{array}$ & $\begin{array}{l}\text { Dilatazioni del tratto urinario sono identificate nell'1-2\% dei feti e possono sottintendere di- } \\
\text { verse nefro-uropatie. Tuttavia, non sempre vi è corrispondenza tra i reperti pre-e post-natali. } \\
\text { Il diametro antero-posteriore della pelvi renale è il parametro che è correlato meglio } \\
\text { con la severità della dilatazione e con la possibilità di risoluzione spontanea della stessa. } \\
\text { È stata, pertanto, messa a punto una classificazione ecografica che permette di strati- } \\
\text { ficare il rischio di presentare una nefro-uropatia. } \\
\text { Sono necessari ulteriori studi per correlare l'imaging pre- e post-natale con la necessità } \\
\text { di eseguire l'intervento chirurgico correttivo e con il rischio di sviluppare un'insufficienza } \\
\text { renale terminale. }\end{array}$ \\
\hline Società di riferimento & Disponibilità online \\
\hline
\end{tabular}

Limitandoci alla considerazione della letteratura pediatrica, più che i diversi articoli epidemiologici che descrivono esperienze più o meno evolute per affrontare la disparità in paesi LMIC, sono rilevanti gli articoli a impronta metodologica, che, a partire dall'analisi di esperienze di cooperazione internazionale $(43,44)$, propongono percorsi (road maps) per un'azione efficace nell'affrontare la CKD pediatrica. Altrettanto importanti, a livello degli HIC, si rivelano le segnalazioni di maggiore mortalità e minore sopravvivenza del rene trapiantato pediatrico in minoranze svantaggiate (40).

Le modalità e le azioni concrete con cui intervenire per colmare il gap delle disuguaglianze sono state oggetto di numerose analisi. Emerge con chiarezza che l'approccio al paziente pediatrico con CKD e alla sua famiglia non può essere che globale, implicando interventi di ordine economico, culturale e sociale.

Data la difficoltà a intervenire su tali fattori, la letteratura recente concorda, quindi, sia sulla necessità di un approccio multidisciplinare, che includa figure sanitarie insieme ad assistenti sociali e di comunità, sia sull'opportunità di un coinvolgimento diretto dei sistemi sanitari nazionali e dei governi (48) nei programmi di cooperazione, congiuntamente a fondazioni private a supporto del pubblico $(43,44)$.

Non si nascondono, comunque, negli articoli più recenti, le molte difficoltà incontrate nel realizzare programmi concreti di intervento e, soprattutto, nel garantirne la sostenibilità.

Nell'ottica di una medicina sempre più globale e rispettosa della persona, il dibattito sugli aspetti etici si mantiene 
vitale, anche nei termini di un'attenzione del singolo medico e delle società scientifiche alla crescita degli interventi a favore dei pazienti svantaggiati, sia nei paesi in via di sviluppo che in quelli industrializzati.

\section{Disclosures}

Conflict of interest: The authors declare no conflict of interest. Financial support: This research received no specific grant from any funding agency in the public, commercial, or not-for-profit sectors.

\section{Bibliografia}

1. van der Ven AT, Vivante A, Hildebrandt F. Novel Insights into the Pathogenesis of Monogenic Congenital Anomalies of the Kidney and Urinary Tract. J Am Soc Nephrol. 2018;29(1):36-50. CrossRef PubMed

2. Hewitt I, Montini G. Vesicoureteral reflux is it important to find? Pediatr Nephrol. 2021;36(4):1011-1017. PubMed

3. Ammenti A, Alberici I, Brugnara M, et al; Italian Society of Pediatric Nephrology. Updated Italian recommendations for the diagnosis, treatment and follow-up of the first febrile urinary tract infection in young children. Acta Paediatr. 2020;109(2):236-247. CrossRef PubMed

4. Jackson AR, Ching CB, McHugh KM, Becknell B. Roles for urothelium in normal and aberrant urinary tract development. Nat Rev Urol. 2020;17(8):459-468. CrossRef PubMed

5. Gordon ZN, McLeod DJ, Becknell B, Bates DG, Alpert SA. Uroepithelial Thickening on Sonography Improves Detection of Vesicoureteral Reflux in Children with First Febrile Urinary Tract Infection. J Urol. 2015;194(4):1074-1079. CrossRef PubMed

6. Gordon ZN, McLeod DJ, Ching CB, et al. Uroepithelial thickening improves detection of vesicoureteral reflux in infants with prenatal hydronephrosis. J Pediatr Urol. 2016;12(4):e251-257. CrossRef

7. Vivarelli M, Massella L, Ruggiero B, Emma F. Minimal Change Disease. Clin J Am Soc Nephrol. 2017;12(2):332-345. CrossRef PubMed

8. Colucci M, Corpetti G, Emma F, Vivarelli M. Immunology of idiopathic nephrotic syndrome. Pediatr Nephrol. 2018; 33(4):573-584. CrossRef PubMed

9. Colucci M, Carsetti R, Cascioli S, et al. B Cell Reconstitution after Rituximab Treatment in Idiopathic Nephrotic Syndrome. J Am Soc Nephrol. 2016;27(6):1811-1822. CrossRef PubMed

10. Trautmann A, Schnaidt S, Lipska-Ziętkiewicz BS, et al; PodoNet Consortium. Long-Term Outcome of SteroidResistant Nephrotic Syndrome in Children. J Am Soc Nephrol. 2017;28(10):3055-3065. CrossRef PubMed

11. Kopp JB, Anders HJ, Susztak K, et al. Podocytopathies. Nat Rev Dis Primers. 2020;6(1):68. CrossRef PubMed

12. Trautmann A, Vivarelli $M$, Samuel $S$, et al; International Pediatric Nephrology Association. IPNA clinical practice recommendations for the diagnosis and management of children with steroid-resistant nephrotic syndrome. Pediatr Nephrol. 2020;35(8):1529-1561. CrossRef PubMed

13. Morello W, Puvinathan S, Puccio G, et al. Post-transplant recurrence of steroid resistant nephrotic syndrome in children: the Italian experience. J Nephrol. 2020;33(4):849-857. CrossRef PubMed

14. Schena FP, Nistor I. Epidemiology of IgA Nephropathy: A Global Perspective. Semin Nephrol. 2018;38(5):435-442. CrossRef PubMed
15. Jarrick $S$, Lundberg $S$, Welander $A$, et al. Mortality in IgA Nephropathy: A Nationwide Population-Based Cohort Study. J Am Soc Nephrol. 2019;30(5):866-876. CrossRef PubMed

16. Fellström BC, Barratt J, Cook H, et al; NEFIGAN Trial Investigators. Targeted-release budesonide versus placebo in patients with IgA nephropathy (NEFIGAN): a double-blind, randomised, placebo-controlled phase 2b trial. Lancet. 2017;389(10084): 2117-2127. CrossRef PubMed

17. Cambier A, Gleeson PJ, Flament H, Le Stang MB, Monteiro RC. New therapeutic perspectives for IgA nephropathy in children. Pediatr Nephrol. 2021;36(3):497-506. CrossRef PubMed

18. Zhang YM, Zhang H. Insights into the Role of Mucosal Immunity in IgA Nephropathy. Clin J Am Soc Nephrol. 2018;13(10): 1584-1586. CrossRef PubMed

19. Zipfel PF, Wiech T, Rudnick R, Afonso S, Person F, Skerka C. Complement Inhibitors in Clinical Trials for Glomerular Diseases. Front Immunol. 2019;10:2166. CrossRef PubMed

20. Fakhouri F, Le Quintrec $M$, Frémeaux-Bacchi V. Practical management of $\mathrm{C} 3$ glomerulopathy and immunoglobulinmediated MPGN: facts and uncertainties. Kidney Int. 2020; 98(5):1135-1148. CrossRef

21. Durey MA, Sinha A, Togarsimalemath SK, Bagga A. Anticomplement-factor $\mathrm{H}$-associated glomerulopathies. Nat Rev Nephrol. 2016;12(9):563-578. CrossRef PubMed

22. Nester CM, Smith RJ. Complement inhibition in C3 glomerulopathy. Semin Immunol. 2016;28(3):241-249. CrossRef PubMed

23. Yamamura T, Horinouchi T, Adachi T, et al. Development of an exon skipping therapy for X-linked Alport syndrome with truncating variants in COL4A5. Nat Commun. 2020;11(1):2777. CrossRef PubMed

24. Cherqui S, Courtoy PJ. The renal Fanconi syndrome in cystinosis: pathogenic insights and therapeutic perspectives. Nat Rev Nephrol. 2017;13(2):115-131. CrossRef PubMed

25. Gabriel SS, Belge H, Gassama A, et al. Bone marrow transplantation improves proximal tubule dysfunction in a mouse model of Dent disease. Kidney Int. 2017;91(4):842-855. CrossRef PubMed

26. Nürnberger J, Philipp T, Witzke O, et al. Eculizumab for atypical hemolytic-uremic syndrome. N Engl J Med. 2009;360(5): 542-544. CrossRef PubMed

27. Ardissino G, Tel F, Sgarbanti M, et al. Complement functional tests for monitoring eculizumab treatment in patients with atypical hemolytic uremic syndrome: an update. Pediatr Nephrol. 2018;33(3):457-461. CrossRef PubMed

28. Schaefer F, Ardissino G, Ariceta G, et al; Global aHUS Registry. Clinical and genetic predictors of atypical hemolytic uremic syndrome phenotype and outcome. Kidney Int. 2018;94(2): 408-418. CrossRef PubMed

29. Ardissino G, Possenti I, Tel F, Testa S, Salardi S, Ladisa V. Discontinuation of eculizumab treatment in atypical hemolytic uremic syndrome: an update. Am J Kidney Dis. 2015;66(1): 172-173. CrossRef PubMed

30. Cuzzoni E, Franca R, De ludicibus S, et al. MIF plasma level as a possible tool to predict steroid responsiveness in children with idiopathic nephrotic syndrome. Eur J Clin Pharmacol. 2019;75(12):1675-1683. CrossRef PubMed

31. Turolo S, Edefonti A, Ghio L, Testa S, Morello W, Montini G. CYP and SXR gene polymorphisms influence in opposite ways acute rejection rate in pediatric patients with renal transplant. $B M C$ Pediatr. 2020;20(1):246. CrossRef PubMed

32. Turolo S, Edefonti A, Lepore M, et al. SXR rs3842689: a prognostic factor for steroid sensitivity or resistance in pediatric idiopathic nephrotic syndrome. Pharmacogenomics. 2016;17(11):1227-1233. CrossRef PubMed 
33. Sahbani D, Strumbo B, Tedeschi S, et al. Functional Study of Novel Bartter's Syndrome Mutations in ClC-Kb and Rescue by the Accessory Subunit Barttin Toward Personalized Medicine. Front Pharmacol. 2020;11:327. CrossRef PubMed

34. Xie Y, Bowe B, Mokdad AH, et al. Analysis of the Global Burden of Disease study highlights the global, regional, and national trends of chronic kidney disease epidemiology from 1990 to 2016. Kidney Int. 2018;94(3):567-581. CrossRef PubMed

35. Harambat J, Ekulu PM. Inequalities in access to pediatric ESRD care: a global health challenge. Pediatr Nephrol. 2016;31(3):353-358. CrossRef PubMed

36. Montini G, Edefonti A, Galán YS, et al. Non-Medical Risk Factors as Avoidable Determinants of Excess Mortality in Children with Chronic Kidney Disease. A Prospective Cohort Study in Nicaragua, a Model Low Income Country. PLoS One. 2016;11(5): e0153963. CrossRef PubMed

37. Harambat J, van Stralen KJ, Verrina E, Groothoff JW, Schaefer F, Jager KJ; ESPN/ERA-EDTA Registry. Likelihood of children with end-stage kidney disease in Europe to live with a functioning kidney transplant is mainly explained by nonmedical factors. Pediatr Nephrol. 2014;29(3):453-459. CrossRef PubMed

38. Ferraz FHRP, Rodrigues CIS, Gatto GC, Sá NM. Differences and inequalities in relation to access to renal replacement therapy in the BRICS countries. Cien Saude Colet. 2017;22(7): 2175-2185. CrossRef PubMed

39. Chesnaye NC, van Stralen KJ, Bonthuis M, Harambat J, Groothoff JW, Jager KJ. Survival in children requiring chronic renal replacement therapy. Pediatr Nephrol. 2018;33(4): 585-594. CrossRef PubMed

40. Patzer RE, Sayed BA, Kutner N, McClellan WM, Amaral S. Racial and ethnic differences in pediatric access to preemptive kidney transplantation in the United States. Am J Transplant. 2013;13(7):1769-1781. CrossRef PubMed

41. Sereni F, Edefonti $A$, Lepore $M$, et al. Social and economic determinants of pediatric health inequalities: the model of chronic kidney disease. Pediatr Res. 2016;79(1-2):159-168. CrossRef PubMed

42. Watkins K. Leaving no one behind: an agenda for equity. Lancet. 2014;384(9961):2248-2255. CrossRef PubMed
43. Edefonti A, Marra G, Castellón Perez M, Sandoval Díaz M, Sereni F; Nicaraguan Network of Pediatric Nephrology (NINEPEN). A comprehensive cooperative project for children with renal diseases in Nicaragua. Clin Nephrol. 2010;74(suppl 1): S119-S125. PubMed

44. Lou-Meda R. ESRD in Guatemala and a model for preventive strategies: outlook of the Guatemalan Foundation for Children with Kidney Diseases. Ren Fail. 2006;28(8):689-691. CrossRef PubMed

45. Pasini A, Benetti E, Conti G, et al. The Italian Society for Pediatric Nephrology (SINePe) consensus document on the management of nephrotic syndrome in children: part I - Diagnosis and treatment of the first episode and the first relapse. Ital J Pediatr. 2017;43(1):41. CrossRef PubMed

46. Trautmann A, Vivarelli $M$, Samuel $S$, et al; International Pediatric Nephrology Association. IPNA clinical practice recommendations for the diagnosis and management of children with steroid-resistant nephrotic syndrome. Pediatr Nephrol. 2020;35(8):1529-1561. CrossRef PubMed

47. Savige J, Gregory M, Gross O, Kashtan C, Ding J, Flinter F. Expert guidelines for the management of Alport syndrome and thin basement membrane nephropathy. J Am Soc Nephrol. 2013;24(3):364-375. CrossRef PubMed

48. Loirat C, Fakhouri F, Ariceta G, et al; HUS International. An international consensus approach to the management of atypical hemolytic uremic syndrome in children. Pediatr Nephrol. 2016;31(1):15-39. CrossRef PubMed

49. Craig BM, Gilbert SM, Herndon JB, Vogel B, Quinn GP. Participation of Older Patients With Prostate Cancer in Medicare Eligible Trials. J Urol. 2010;184(3):1134-1144. CrossRef PubMed

50. Nguyen HT, Benson CB, Bromley B, et al. Multidisciplinary consensus on the classification of prenatal and postnatal urinary tract dilation (UTD classification system). J Pediatr Urol. 2014;10(6):982-998. CrossRef PubMed

51. Farrugia MK, Hitchcock $R$, Radford $A$, Burki $T$, Robb $A$ Murphy F; British Association of Paediatric Urologists. British Association of Paediatric Urologists consensus statement on the management of the primary obstructive megaureter. J Pediatr Urol. 2014;10(1):26-33. CrossRef PubMed 